\title{
ANTIMICROBIAL UTILIZATION PATTERN IN A TEACHING DISTRICT HOSPITAL OF NEPAL
}

\author{
Das B P*, Sethi A*, Rauniar G P
}

\section{ABSTRACT}

The objective of this study was to monitor the antimicrobial utilization in a teaching district hospital and to compare it against the rational antimicrobial use guidelines.

Total 106 data sheet of indoor patients admitted in year 2001 were randomly chosen and analyzed. The number of antimicrobials prescribed in every prescription was taken into account to calculate the incidence of use of more than one antimicrobial agent. The Patient sheet included patient information, drug information (name, indication, dose, frequency and duration of antimicrobial use), diagnosis or provisional diagnosis and investigations. Prescription auditing was done on the selected prescriptions.

Male: female ratio was 40:65. In 90.57\% diagnosis was established empirically and in only $4.7 \%$ patients confirmatory diagnosis was made using relevant investigations. The antimicrobials were prescribed chiefly for respiratory tract infections (RTI -26.41\%), gastrointestinal infections (GIT -22.64\%), genital tract infection (13.20\%), wound infections $(\mathbf{8 . 4 8 \%})$, urinary tract infections (UTI-6.6\%), bone $\&$ joint infections $(4.71 \%)$ and miscellaneous (Typhoid, Malaria, meningitis etc-17.92\%). The causative organism was confirmed using culture $\&$ sensitivity tests in only one patient $(0.94 \%)$ out of 106 patients. In $77(69.8 \%)$ patient prescriptions suspected pathogenic organisms were mentioned. These were streptococcus (29.58\%), Chlamydia (11.27\%), mycoplasma (18.30\%), staphylococcus $(23.94 \%)$, E. coli $(32.39 \%)$ and S. typhi (15.49\%). In 35 prescriptions (33.01) no causative organism was mentioned. It was further observed that a total 452 drugs (i.e. 4.26 drugs per patient), out of this only 165 antimicrobials (1.55 antimicrobials per patient) were prescribed in the 106 enrolled prescriptions. On further analysis of antimicrobials use, it was observed that most patients were prescribed a single antimicrobial agent (58.49\%), two AMAs (24.52\%), three AMAs (13.20\%) and least was four AMAs (3.77\%).The antimicrobials were given prophylactically in $14(\mathbf{1 3 . 1 0 \%})$ and therapeutically in $85(\mathbf{8 0 . 1 8 \%})$ prescriptions. The use of antimicrobials were not indicated in $\mathbf{7}(6.60 \%)$ prescriptions.. In 91 cases $(\mathbf{8 5 . 8 5 \%})$ duration for which AMAs were considered was not mentioned. Ampicillin + cloxacillin $(12.12 \%)$, ciprofloxacin $(10.30 \%)$, metronidazole $(10.30 \%)$, amoxycillin (10.30\%) and cephalosporins $(\mathbf{2 5 . 4 7 \%})$ were the most frequently prescribed AMAs. A fixed dose combination of ampicillin \& cloxacillin, ciprofloxacin and metronidazole were recurrently prescribed while gentamicin, cotrimoxazole and crystalline penicillin were relatively least prescribed antimicrobials agents.

Inappropriate and irrationality in the Antimicrobial prescribing pattern was observed.

Key Words: Antimicrobial agent, Respiratory tract infection (RTI), urinary tract infection (UTI), gastrointestinal infection, prophylaxis, therapeutic.

${ }^{*}$ B.P. Koirala Institute of Health Sciences, Dharan, Nepal.

Address for correspondence : Dr. B. P. Das

B.P. Koirala Institute of Health Sciences, Dharan, Nepal.

Email: bpdas2000@yahoo.com 


\section{INTRODUCTION}

The management of infectious diseases has been revolutionized by the use of antimicrobial agents (AMA). However the intensive use of these agents has led to the development of bacterial resistance, increased incidence of adverse effects, drug-drug interactions \& increase the cost of medical care for the patient. Moreover the problem of inappropriate use of AMA is recognized as a major health hazard world wide. Therefore, intensive drug utilization evaluation is necessary for monitoring these events. ${ }^{1}$ The importance of studying prescribing behavior have been demonstrated by a number of reports. ${ }^{2-4}$ The survey conducted in China found that drug fees accounted for $72-83 \%$ of average charge per outpatient visit. ${ }^{5}$ The National Health Services Survey (NHSS) reviewed that township health centers earned $69 \% \& 77 \%$ of their total income from only the sales of drug in the year 1986 and $1992,{ }^{6}$ thus the indiscriminate use of antimicrobials is burdening the cost of health care. Despite the prevalent use of antimicrobials in community practice, the use in this setting is accounted inappropriate and irrational. Wyatt et $\mathrm{al}^{7}$ showed the use of amoxicillin, ampicillin, Penicillin $\mathrm{V}$ and erythromycin accounted for the $70 \%$ of AMA in community use of oral antimicrobials dispensed empirically in Northern Ireland from 1983 to 1987.

Only a few studies are available where the antimicrobials are prescribed rationally. Mc Caig \& Hughes ${ }^{8}$ reported otitis media, upper respiratory tract infection, bronchitis, pharyngitis \& sinusitis, which are mostly nonbacterial in origin were the leading indications in $76 \%$ of total antimicrobial prescriptions and the antimicrobials used were amoxicillin, cephalosporias, penicillin, erythromycin and cotrimaoxazole. Mc Manus et $\mathrm{al}^{9}$ showed that the most commonly prescribed AMAS were cefaclor, amoxicillin \& a combination of amoxicillin and clauvulanic acid in Australia. Kentucky (US) and Mainous et a $1^{10}$ observed that antimicrobials (primarily amoxicillin, second /third generation cephalosporins \& erythromycin) were prescribed in $60 \%$ of patients suffering from common cold.

Thus, it is important to evaluate and monitor antimicrobials utilization in the different geographical areas as there is a lot of variation in the superlation of antimicrobials by the physicians.

As, these studies are not conducted previously in district hospitals of Nepal till date. BP Koirala Institute of Health Sciences has adopted seven district hospitals of eastern Nepal for internship training program, as a periphery posting supervised by the faculty member of this institute. Hence, this study was a modest endeavor to observe antimicrobials utilization pattern in a teaching district hospital in Nepal. This would also help to formulate a base line data that would advice the hospital authorities to promote rational use of AMAs at various district hospitals in Nepal.

\section{MATERIALS AND METHODS}

Total 106 data sheet of indoor patients admitted in year 2001 in Dhankuttta district hospital were randomly chosen and analyzed. The number of antimicrobials prescribed in every prescription was taken into account to calculate the incidence of use of more that one antimicrobial agent. The Patient sheet included patient information, drug information (name, indication, dose, frequency and duration of antimicrobial use), diagnosis or provisional diagnosis and investigations. Prescription auditing was done on the selected prescriptions by the author.

\section{RESULTS}

Total 106 prescriptions of indoor patients were scrutinized for various age distributions and sex ratio were analyzed (Table I). In $90.57 \%$ diagnosis was established empirically

Table I : Age, Sex distribution of Patient $(n=106)$

\begin{tabular}{l|l}
\hline Patient & Number $(\%)$ \\
\hline$\underline{\text { Age }}$ & \\
0 -10 years & $11(10.37)$ \\
11-20 years & $28(26.41)$ \\
21-30 years & $19(17.92)$ \\
31-50 years & $17(16.03)$ \\
$51-60$ years & $13(12.26)$ \\
$>60$ years & $18(16.98)$ \\
Not mentioned & $4(3.77)$ \\
& \\
Sex & \\
Male & $40(37.73)$ \\
Female & $65(61.32)$ \\
Not mentioned & $1(0.94)$ \\
\hline
\end{tabular}




\begin{tabular}{|c|c|c|c|}
\hline \multicolumn{4}{|c|}{ Table II(A) : Basis of diagnosis } \\
\hline S. No. & Basis & \multicolumn{2}{|c|}{ Number $(\%)$} \\
\hline 1. & Clinical & \multicolumn{2}{|c|}{$96(90.57)$} \\
\hline 2. & Laboratory & \multicolumn{2}{|c|}{$1(0.94)$} \\
\hline 3. & Radiology & \multicolumn{2}{|c|}{$2(1.89)$} \\
\hline 4. & Clinical + Laboratory & \multicolumn{2}{|c|}{$3(2.83)$} \\
\hline 5. & Clinical + Radiology & \multicolumn{2}{|c|}{$2(1.89)$} \\
\hline 6. & Not mentioned & \multicolumn{2}{|c|}{$2(1.89)$} \\
\hline \multicolumn{4}{|c|}{ Table II(B) : Diagnosis (Disease pattern) $n=106$} \\
\hline S. No. & \multicolumn{2}{|l|}{ Diagnosis } & Number (\%) \\
\hline 1. & \multicolumn{2}{|l|}{ RTI } & $28(26.41)$ \\
\hline 2. & \multicolumn{2}{|l|}{ GIT } & $24(22.64)$ \\
\hline 3. & \multicolumn{2}{|l|}{ Genital tract } & $14(13.20)$ \\
\hline 4. & \multicolumn{2}{|l|}{ Injury and ulcer } & $9(8.49)$ \\
\hline 5. & \multicolumn{2}{|l|}{ UTI } & $7(6.60)$ \\
\hline 6. & \multicolumn{2}{|l|}{ Bone \& Joint infection } & $5(4.71)$ \\
\hline 7. & \multicolumn{2}{|c|}{ Others (Typhoid, Malaria meningitis etc.) } & $19(17.92)$ \\
\hline \multicolumn{4}{|c|}{ Table III : Bacteriological data } \\
\hline \multicolumn{4}{|c|}{ Details of bacteriological investigation } \\
\hline \multicolumn{2}{|l|}{ Yes } & \multicolumn{2}{|r|}{$1(0.94)$} \\
\hline \multicolumn{2}{|l|}{ No } & \multicolumn{2}{|r|}{$101(95.28)$} \\
\hline \multicolumn{2}{|c|}{ Not mentioned } & \multicolumn{2}{|r|}{$4(3.77)$} \\
\hline \multicolumn{2}{|c|}{$\underline{\text { Suspected organisms }}$} & & \\
\hline \multicolumn{2}{|c|}{ Mentioned } & \multicolumn{2}{|r|}{$71(6.98)$} \\
\hline \multicolumn{2}{|c|}{ Not mentioned } & \multicolumn{2}{|r|}{$35(33.01)$} \\
\hline \multicolumn{2}{|c|}{ Commonly suspected bacteria (n- 71) } & & \\
\hline Streptoco & & & $21(29.58)$ \\
\hline Chlamydi & & & $8(11.27)$ \\
\hline Mycopla: & & & $13(18.30)$ \\
\hline Staphyloc & & & $17(23.94)$ \\
\hline E. coli & & & $23(32.39)$ \\
\hline S. typhi & & & $11(15.49)$ \\
\hline
\end{tabular}

INMAA Mav -.lıne 20ก4 4.3 
Table III : Antimicrobials (AMAs) data (n=106)

\begin{tabular}{l|l}
\hline Total no. of drugs & $452($ mean -4.26$)$ \\
\hline Total no of antimicrobials agent & $165($ mean -1.55$)$ \\
No. of drugs/ prescription $(\mathbf{n}=\mathbf{1 0 6})$ & \\
\hline 2 drugs & $4(3.78)$ \\
3 drugs & $33(31.13)$ \\
4 drugs & $28(26.41)$ \\
5 drugs & $17(16.03)$ \\
6 drugs & $13(12.26)$ \\
7 drugs & $6(5.66)$ \\
9 drugs & $5(4.71)$ \\
\hline
\end{tabular}

Table IV : Antimicrobial Indication and Duration

\begin{tabular}{l|l}
\hline No. of AMAs / prescription & $62(58.49)$ \\
\hline 1 drug & $26(24.52)$ \\
2 drugs & $14(13.20)$ \\
3 drugs & $4(3.77)$ \\
Nil & $14(13.10)$ \\
\hline Basis of use of AMAs & $85(80.18)$ \\
Proplylaxis & $7(6.60)$ \\
Nherapeutic & $1(0.94$ \\
\hline Duration of use of AMAs & $10(9.43)$ \\
\hline 48 hrs & $91(85.85)$ \\
\hline Not mentioned & \\
\hline
\end{tabular}

Table V : Ten Commonly used AMAs

\begin{tabular}{l|ll}
\hline 1. & Cephalosporin & $27(15.47)$ \\
2. & Ampiclillin + cloxacillin & $20(12.12)$ \\
3. & Ciprofloxacin & $17(10.30)$ \\
4. & Metronidazole & $17(10.30)$ \\
5. & Amoxycillin & $17(10.30)$ \\
6. & Chloramphenicol & $13(7.87)$ \\
7. & Erythromycin & $11(6.66)$ \\
8. & Gentamycin & $8(4.84)$ \\
9. & Crystalline penicillin & $6(3.63)$ \\
10. & Others & $32(19.36)$ \\
\hline
\end{tabular}

and in only $4.7 \%$ patients (using both clinical examination with radiological $(1.89 \%)$ or bacteriological examination $(2.83 \%)$, confirmatory diagnosis was built. (Table II). The antimicrobials were prescribed for respiratory tract infections (RTI -26.41\%), gastrointestinal infections (GIT -22.64\%), genital tract infection (13.20\%), wound infections (8.48\%), urinary tract infections (UTI-6.6\%), bone \& joint infections $(4.71 \%)$ and miscellaneous (Typhoid, Malaria, meningitis etc-17.92\%). The causative organism was confirmed using culture \& sensitivity tests in only one patient $(0.94 \%)$ out of 106 patients (Table III). In 77 (69.8\%) patient prescriptions the suspectible pathogenic organisms were mentioned by the physician in the antibiotic audit questionnaire supplemented with the data sheet of patients. These were streptococcus (29.58\%), Chlamydia (11.27\%), mycoplasma (18.30\%), staphylococcus (23.94\%), E. coli (32.39\%) and S. typhi (15.49\%) (Table III). In 35 prescriptions, no caustive organism was mentioned. It was further observed that a total 
452 drugs (i.e. 4.26 drugs per patient) were dispensed, out of this only 165 antimicrobials (1.55 antimicrobials per patient) were prescribed to the patients (Table IV \& V). On further analysis of antimicrobials use, it was observed that most patients were prescribed a single antimicrobial agent (58.49\%), two AMAs $(24.52 \%)$, three AMAs (13.20\%) and least was four AMAs (3.77\%) (Table IV \& V).

The therapeutic or the prophylactic use of antimicrobials were decided empirically by the physician on clinical judgement of the patient. The antimicrobials were given prophylactically in $14(13.10 \%)$ and therapeutically in $85(80.18 \%)$ prescriptions. The use of antimicrobials were not indicated in $7(6.60 \%)$ prescriptions. In $85.85 \%$ of the patients, duration for which AMAs were considered was not mentioned. Ampicillin + cloxacillin (12.12\%), ciprofloxacin (10.30\%), metronidazole $(10.30 \%)$, amoxycillin (10.30\%) and cephalosporins mainly cefadroxilor cephalexin $(25.47 \%)$ were the most frequently prescribed AMAs. A fixed dose combination of ampicillin \& cloxacillin, ciprofloxacin and metronidazole were recurrently prescribed while gentamicin, cotrimoxazole and crystalline penicillin were relatively least prescribed antimicrobials agents (Table V).

\section{DISCUSSION}

The study of prescribing pattern is an essential component of medical audit that seeks monitoring, evaluation and necessary modifications in the prescribing practices of prescribers to achieve rational and cost effective medical care for the patient Antimicrobials are a major part of hospital pharmacy budgets and must be considered in resource planning and spending projections. Setting standards and assessing the quality of AMA utilization through performance and review should be a part of routine clinical practice. ${ }^{11}$ Logically, trends in antimicrobial usage should be linked to sensitivity pattern of microorganisms. Auditing of prescriptions also form a part of drug utilization studies. ${ }^{12}$ Average number of drugs per prescription is an important index of prescription audit. It is preferable to keep the mean number of drugs per prescription as low as possible since higher figures always lead to increase risk of drug interaction, ${ }^{13}$ development of bacterial resistance and increased hospital costs. ${ }^{14}$ This study documents the extent of antimicrobial use in medical service in a rural district hospital and the prescribing behavior of the qualified district hospital doctors.

In our study, the prescribing frequency of antimicrobials per prescription is mostly one $(58.49 \%)$ or two $(24.52 \%)$, this would curtail the cost of treatment as well as the incidence of development of adverse drug reactions in patients. Fixed

dose combination of ampicillin \& cloxacillin and ciprofloxacin with metronidazole or amoxycillin were most habitually prescribed antimicrobial drugs. Mostly AMA are used empirically on the basis of clinical diagnosis (90.57\%). The duration of AMA were unavailable in $86 \%$ of prescriptions.. The bacteriological confirmation was done in only one patient. The patients were prescribed irrationally for UTI or GIT infections most of which are viral in origin and if the cause is bacterial, it should be ascertained before instituting antimicrobials. Thus, our study indicates the irrational use of AMAs in this district hospital of Dhankuta.

In developing country like Nepal, patient compliance is primarily dependent on the cost of treatment. Among the AMAs reviewed, quinolones and cephalosporins are relatively expensive drugs \& thus unaffordable for the patient particularly in the rural area. Most of the patients were given AMAs on the basis of clinical diagnosis and confirmatory diagnosis using relevant investigation was seldom applied. This could be explained due to limited facilities for investigations at that center. As, the antimicrobials have a limited spectrum against pathogens, culture \& sensitivity is usually recommended for the safe and rationale use of AMAs. It was observed that culture and sensitivity was performed in only one patient which is incorrect in view of the rational use of AMAs.

The indication for AMA use was not cited in 35 (33.01\%) prescriptions; this depicts the incongruous use of AMAs. 1.55 antimicrobials per patient were prescribed, this finding states that antimicrobials were started in most of the patients without making an attempt to identify the etiological agents. This prescribing behavior depicts that despite implementation of antimicrobial guidelines formulated by the various international bodies, there is prevalence of irrational AMA use. Hence, there is an urge for more studies to monitor effectivity of antimicrobial control programmes and implementation of training sessions for medical personnel to rationalize their attitude for a good prescribing.

This observation carries a negative impact on the training of the interns of B.P. Koirala Institute of Health Sciences. However, the use of low cost AMAs at district hospital level can decrease the cost and improve patient compliance. Limited use of AMAs also decreases the chance of resistance. The prescribing of expensive AMAs without microbiological confirmation should have been avoided wherever possible, especially when other alternative AMAs were available.

The B.P Koirala institute of Health Sciences is planing to establish the guidelines for the empirical use of antimicrobials. The proposal is in the pipeline., this would assist clinicians with the appropriate selection of prophylactic and empiric 
antibiotic therapy for potential and common infections seen at the Medical Center. Antibiotic guidelines are by no means a replacement for good clinical judgement. Rather, these guidelines are to be used as a guide when considering the initiation of antibiotic therapy. and would promote rational \& cost effective use of AMAs .Finally, they serve as drug use evaluation criteria for anti-infective audits. Regular prescription audit with feedback to prescribers, training programmes to health workers in district level and community health education programmes will help to ensure safe and rational use of AMAs in district hospitals.

\section{REFERENCE}

1. Stratton CL, Ratner H, Johston PE, Schaffner W. Focused microbiologic surveillance by specific hospital unit: Practical application and clinical utility. Clin Ther 1993: 115 (Suppl A) ; $12-20$

2. Gilson L, Jaffar S. Mwankusye S, Teuscher T. Assessing prescribing practice; a Tanzanian example. Int J Health planning Management 1993: 8: 27-58.

3. Naja SA, Idris MZ, Khan A. Drug cost more at primary health clinics: an experience from Libya. Health Policy Planning 1988; 3: 69-73.

4. Mnyika KS, Killewo JZJ. Irrational drug use in Tanzania. Health Policy planning 1991; 6: 180-4.

5. Bloom G, Lucas H, Cao S, Yao J, Gao J, Gu X. et al financing health services in poor rural areas: adopting to economic and institutional reform in China. In : IDS Research Report, No. 30. Brighton: Institute of development studies, 1995.

6. Ministry of public health research on National Health Services, Beijing: MopH, 1994.

7. Wyatt TD, Passmore CM, Morrow NC, et al. Antibiotic prescribing: the need for a policy in general practice. $B M U$ 1990: 300: 441-4.

8. MC Caig LF, Hughes JM. Trends in antimicrobial drug prescribing among office based physicians in the United States. JAMA 1995: 273: 214-19.

9. Mc Manus $P$, Hammond ML, Whicker SD, et al. Antibiotic use in the Australian Community, 1990 - 1995. Med J Aus. 1997: 167: $124-7$.

10. Mainous AG, Hueston WJ, Clark JR. Antibiotics and Upper respiratory infection. Do some folks think there is a cure for the common cold. J Fam Pract 1996: 42: 357-61.

11. Patterson HR. The problems of audit and research J Coll Gen pract 1986: 36: 196.

12. WHO Regional publications. Studies in drug utilization. European series No. 8 copenhagen : WHO regional publication, 1979.

13. Niles SA. Principles of therapeutics. In : gilman GA, Rall WT, Nies SA, Taylor $\mathrm{P}$ (eds.). The pharmacological basis of therapeutics $8^{\text {th }}$ edn. New York: pergamon press, 1990: 6283.

14. Atanasova I, Terziivanov D. Investigations on antibiotics in a hospital for a one year period. Int J clin Pharm Ther 1995: 33: 32-3.

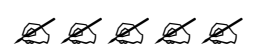

